\title{
Slow shock interactions in the heliosphere using an adaptive grid MHD model
}

\author{
C.-C. $\mathrm{Wu}^{1}$, M. Dryer ${ }^{2,3}$, and S. T. Wu ${ }^{1}$ \\ ${ }^{1}$ CSPAR/The University of Alabama in Huntsville, Huntsville, AL 35899, USA \\ ${ }^{2}$ Exploration Physics International, Inc., Huntsville, AL 35806, USA \\ ${ }^{3}$ NOAA Space Environment Center, Boulder, CO 80305, USA
}

Received: 19 April 2004 - Revised: 3 December 2004 - Accepted: 8 December 2004 - Published: 30 March 2005

\begin{abstract}
A one-dimensional (1-D), time-dependent, adaptive-grid MHD model with solar wind structure has been used in the past to study the interaction of shocks. In the present study, we wish to study some fundamental processes that may be associated with slow shock genesis and their possible interactions with other discontinuities. This adaptivegrid model, suitable for appropriate spatial and temporal numerical simulations, is used for this purpose because its finer grid sizes in the vicinity of the steep gradients at shocks make it possible to delineate the physical parameters on both sides of the shocks. We found that a perturbation with deceleration of solar wind will generate an ensemble consisting of a forward slow shock, a fast forward wave and a reverse slow shock. On the other hand, a perturbation with an increase in acceleration of solar wind will generate both a slow shock and a fast shock. These two perturbations, although not unique, may be representative of momentum and pressure changes at the solar surface.

During the transition of a fast shock overtaking a slow shock from behind, the slow shock might disappear temporarily. Also, during the process of the merging of two slow shocks, a slow shock-like structure is formed first; later, the slow shock-like structure evolves into an intermediate shock-like structure. This intermediate shock-like structure then evolves into an intermediate wave and a slow shock-like structure. Finally, the slow shock-like structure evolves into a slow shock, but the intermediate wave disappears by interacting with the non-uniform solar wind. This complex behavior demonstrates the non-unique nature of the formation of slow shocks, intermediate shocks and their derivative structures.

We emphasize the main aim of this work to be both: (a) non-unique input physical parameters to explain the paucity of observed slow shocks, as well as (b) the impossibility of backward tracing to the history of input boundary conditions in view of the present inability to describe unambiguous inputs at the Sun.
\end{abstract}

Correspondence to: $\mathrm{C} .-\mathrm{C} . \mathrm{Wu}$

(wuc@cspar.uah.edu)
Keywords. Interplanetary physics (Interplanetary shocks, Interplanetary magnetic fields, MHD waves and turbulence)

\section{Introduction}

There are six classical shock solutions - one fast and one slow shock, and four intermediate shocks (DeHoffmann and Teller, 1950), according to the theory of magnetohydrodynamic (MHD) Rankine-Hugoniot relations. All six shocks are coplanar: the upstream and downstream magnetic field and velocity vectors are in the same plane. Fast shocks produce a jump from the upstream flow speed from above the fast speed to below the fast speed downstream; also the transverse component of the magnetic field increases across the fast shock. Slow shocks change the flow speed from above the slow speed upstream to below the slow speed downstream, and the transverse component of the magnetic field decreases across the slow shock. Intermediate shocks take the flow speed from above the intermediate speed upstream to below the intermediate speed downstream; in addition, they rotate the transverse component of the magnetic field by $180^{\circ}$ (Kennel et al., 1989). From simulation results, Chu and Taussig (1967) showed that the intermediate shock is nonevolutionary, and claimed that the intermediate shocks should not be expected in nature. However, Wu (1987) showed that, via simulation of a set of resistive MHD equations, that the intermediate shock can be stable in some situations. In addition, Chao et al. (1993) identified an intermediate shock near $9 \mathrm{AU}$ from the Voyager 1 plasma and magnetic field data.

Whang (1987) suggested that the decrease in the Alfvén speed at increasing heliocentric distance causes the normal Alfvén Mach number of a forward slow shock to become greater than 1, and the shock should eventually evolve from a slow shock into a fast shock. Chao et al. (1987) and Wu et al. (1996a) employed two ideal finite-difference MHD simulation codes to simulate a slow forward shock propagating in a non-uniform solar wind. The simulation demonstrated that 
the speed of the slow shock decreases and that the slow shock does not evolve into a fast shock in a special situation that involves propagation into a positive density gradient.

In this paper, we use a one-dimensional, time-dependent, adaptive-grid, MHD model with a representative solar wind structure to study the interaction of slow shocks. Actually, the model is a $1 \frac{1}{2} \mathrm{D}$ code in the sense that the transverse velocity and magnetic field components are considered; however, their variation with respect to the azimuthal direction is taken to be zero. Three cases with different types of perturbation are studied in this paper. In Case 1, we will show that a forward slow shock (FSS) and a reverse slow shock (RSS) can be generated by a perturbation that incorporates a decreasing pressure and radial velocity. In Case 2, a perturbation with increasing pressure and radial velocity will generate both a slow shock and a fast shock.

In order to understand further the interaction between the fast and slow shocks, two perturbations will be put into the lower boundary (at $28 R_{\odot}$, where $R_{\odot}$ is the solar radius, $6.95 \times 10^{5} \mathrm{~km}$ ) at a different time period for Case 3. First, we introduce a perturbation with decreasing pressure and radial velocity at the lower boundary in order to generate slow shocks. Later, we introduce another perturbation with increasing pressure and radial velocity at the lower boundary, in order to generate a fast shock and allow it to overtake a slow shock from behind. The results will show that the slow shock disappears temporarily during the transition of the fast shock overtaking the slow shock from behind; during the temporal transition of the two merging slow shocks, a slow shock-like structure will first be created. The slow shocklike structure will then evolve into an intermediate shock-like structure later. Then, the intermediate shock-like structure will evolve into a slow shock-like structure and an intermediate wave. The former will steepen into a slow shock, but the latter will disappear by interacting with the non-uniform solar wind.

The main aim of this work is to explore two points via a limited search in input parameter space. The first point is to examine some possible reason(s) for the paucity of observed slow shocks. The second point is to explore the possibility of backward tracing of various interplanetary discontinuities to the history of input boundary conditions at the Sun. We are not aware of published work in either of these two contexts. The parameter space for this objective is almost limitless. Nevertheless, we have chosen a limited and a specific set of momentum and thermal pulses that might represent activity within active regions or coronal holes. We do not consider changes in the magnetic field in this study.

The basic MHD equations are listed in the next section entitled "Magnetohydrodynamic Equations." We will discuss in more detail the interaction of two slow shocks and the evolution of slow shock-like and intermediate shock-like structures in a non-uniform solar wind in Sect. 3 on "Simulations Results." A discussion and summary of our results are given in the final section.

\section{Magnetohydrodynamics Equations}

The large-scale dynamics in the interplanetary solar wind can be theoretically modeled by the following MHD equations (Priest, 1982; Hughes and Brighton, 1967) in SI units,

$$
\begin{aligned}
& \frac{D \rho}{D t}+\rho \nabla \cdot \mathbf{V}=0 \\
& \rho \frac{D \mathbf{V}}{D t}=-\nabla p+\frac{1}{\mu_{\circ}}(\nabla \times \mathbf{B}) \times \mathbf{B}-\rho \frac{G M(r)}{r^{2}} \hat{\mathbf{r}} \\
& \frac{\partial}{\partial t}\left[\rho e+\frac{1}{2} \rho|\mathbf{V}|^{2}+\frac{|\mathbf{B}|^{2}}{2 \mu_{\circ}}\right]+\nabla \cdot\left[\mathbf{V}\left\{\rho e+\frac{1}{2} \rho|\mathbf{V}|^{2}+p\right\}\right. \\
& \left.\quad+\frac{\mathbf{B} \times(\mathbf{V} \times \mathbf{B})}{\mu_{\circ}}\right]=-\mathbf{V} \cdot \rho \frac{G M(r)}{r^{2}} \hat{\mathbf{r}} \\
& \frac{\partial \mathbf{B}}{\partial t}=\nabla \times(\mathbf{V} \times \mathbf{B})
\end{aligned}
$$

where $\frac{D}{D t}$ denotes the total derivative, $\rho$ is the mass density, $\mathbf{V}$ is the velocity of the flow, $p$ is the isotropic gas pressure, $G M(r)$ is the gravitation force, $\mathbf{B}$ is the magnetic field, $e$ is the internal energy per unit mass $(e=p /(\gamma-1) \rho)$ and $\gamma$ is the specific heat ratio. Equations (1), (2) and (3) express the conservation of mass, momentum and energy, respectively. In Eq. (2), we have ignored the viscous term but included the gravitational force. Equation (4) is the induction equation for a perfectly conducting medium. The formulation is then closed by the ideal gas law $p=2 \rho R T$ and the divergence free magnetic field, $\nabla \cdot \mathbf{B}=0$. Here $R$ is the gas constant; $T$ is the isotropic plasma temperature; and a factor of 2 is due to the fully ionized nature of the hydrogen solar wind plasma.

\section{Simulation Results}

In the following, the transition of interaction of slow shocks will be presented in detail. The numerical scheme used in this analysis is an extension of the two-step Lax-Wendroff finite difference method (Lax and Wendroff, 1960). An adaptive grid finite difference scheme (Panitchob, 1987; Panitchob et al., 1987) is used here to understand the particularly interesting cases of the interaction of slow shocks. The simulation domain is between $28 \mathrm{R}_{\odot}$ and $230 \mathrm{R}_{\odot}$. The reason we chose the lower boundary to be at $28 \mathrm{R}_{\odot}$ is that the solar wind is already supersonic and super-Alfvénic there; hence, the perturbation can be chosen arbitrarily at the lower boundary (Wu and Wang, 1987). The steady-state representative solar wind is obtained here from Panitchob's (1987) non-relativistic MHD solar wind model. Table 1 summarizes the steady-state values at $28 \mathrm{R}_{\odot}$ and $1 \mathrm{AU}$. Figure 1 (from Wu et al., 1996b) shows a plot of the physical parameters of the steady-state solar wind. This calculation is sometimes referred to as a $1 \frac{1}{2} \mathrm{D}$ simulation, where only spatial gradients in the radial direction of the two vector components of velocity and magnetic field are considered. According to earlier simulation results (Wu, 1993; Wu et al., 1996b), a negative, nearly-square wave pulse with decreasing density, temperature or radial velocity will create a pair of slow shocks 
Table 1. Summary of steady-state solar wind values at $28 R_{\odot}$ and $1 \mathrm{AU}$.

\begin{tabular}{ccc}
\hline Dependent Variable & $\begin{array}{c}\text { Steady-State Value } \\
\text { At } 28 R_{\odot}\end{array}$ & $\begin{array}{c}\text { Steady-State Value } \\
\text { At } 1 \mathrm{AU}\end{array}$ \\
\hline $\mathrm{T}, \mathrm{K}$ & $3.7684 \times 10^{5}$ & $1.3 \times 10^{5}$ \\
$\rho, \mathrm{gm} / \mathrm{cm}^{3}$ & $1.026 \times 10^{-21}$ & $1.453 \times 10^{-23}$ \\
$V_{r}, \mathrm{~km} / \mathrm{s}$ & 261.28 & 312.890 \\
$V_{\phi}, \mathrm{km} / \mathrm{s}$ & 4.0012 & 1.263 \\
$B_{r}$, gauss & $2.948 \times 10^{-3}$ & $5 \times 10^{-5}$ \\
$B_{\phi}$, gauss & $-6.1357 \times 10^{-4}$ & $-7.143 \times 10^{-5}$ \\
$\gamma:$ Polytropic Index & 1.25 & 1.25 \\
\hline
\end{tabular}

(a forward and a reverse slow shock). Also, a nearly-square wave pulse with increasing density, temperature or radial velocity will create a pair of slow shocks and a pair of fast shocks (a forward fast shock and a reverse fast shock).

In order to generate these shock ensembles, we introduce a perturbation at the lower boundary with respect to the steady solar wind condition, as given in Fig. 1. Two specific simulations are studied. Case 1 involves a decrease in both radial velocity and pressure; Case 2, an increase of these parameters.

In the first case, we put a perturbation with $\frac{v_{r}^{\prime}}{v_{r_{0}}}=0.4$ and $\frac{T^{\prime}}{T_{\circ}}=0.4$ at the lower boundary, where $v_{r_{\circ}}, T_{\circ}$ represent the initial value of radial velocity and temperature at $28 \mathrm{R}_{\odot} ; v_{r}^{\prime}$ and $T^{\prime}$ represent the temporal evolution of density and temperature at $28 \mathrm{R}_{\odot}$. The rise and decay time of the disturbance is one hour, and the total duration time is $9 \mathrm{~h}$. At $\mathrm{t}=15 \mathrm{~h}$, a reverse slow shock (RSS) and a forward slow shock (FSS) are generated, propagating toward Earth (see Fig. 2). This ensemble follows behind a forward fast wave (FFW) which is seen at $\mathrm{r}=57 R_{\odot}$ at $\mathrm{t}=15 \mathrm{~h}$ (see Fig. 2a). At $\mathrm{t}=97 \mathrm{~h}$, this expanding ensemble propagates outward while the sunward properties recover to their original conditions, as indicated in Fig. 2 b. These results are a repetition of those from $\mathrm{Wu}$ et al. (1996b).

In the second case, we initialized a perturbation at $28 \mathrm{R}_{\odot}$ with $\frac{v_{r}^{\prime}}{v_{r_{0}}}=1.6$ and $\frac{T^{\prime}}{T_{0}}=5$. At $\mathrm{t}=5 \mathrm{~h}$, a forward fast shock (FFS) was formed at $40.1 \mathrm{R}_{\odot}$ and an FSS was formed at $38.6 \mathrm{R}_{\odot}$ both continuing to propagate toward the Earth (see Fig. 3). Note that a substantial length of time $(\sim 77 \mathrm{~h})$ is required before the sunward side of the FSS relaxes to its original set of properties. This is a result of the relaxation time of the added kinetic and thermal energy to be distributed to the outer heliosphere. Figures $3 \mathrm{~b}$ and $3 \mathrm{c}$ show the development, respectively, at $\mathrm{t}=11 \mathrm{~h}$ and $\mathrm{t}=77 \mathrm{~h}$. Note that the FSS decays into a forward slow wave (see Fig. 3c).

Many fast mode MHD shocks have been observed by in-situ spacecraft, but only a few limited number of slow shocks have been observed at heliocentric distances greater than $0.3 \mathrm{AU}$. In order to understand why only a few limited

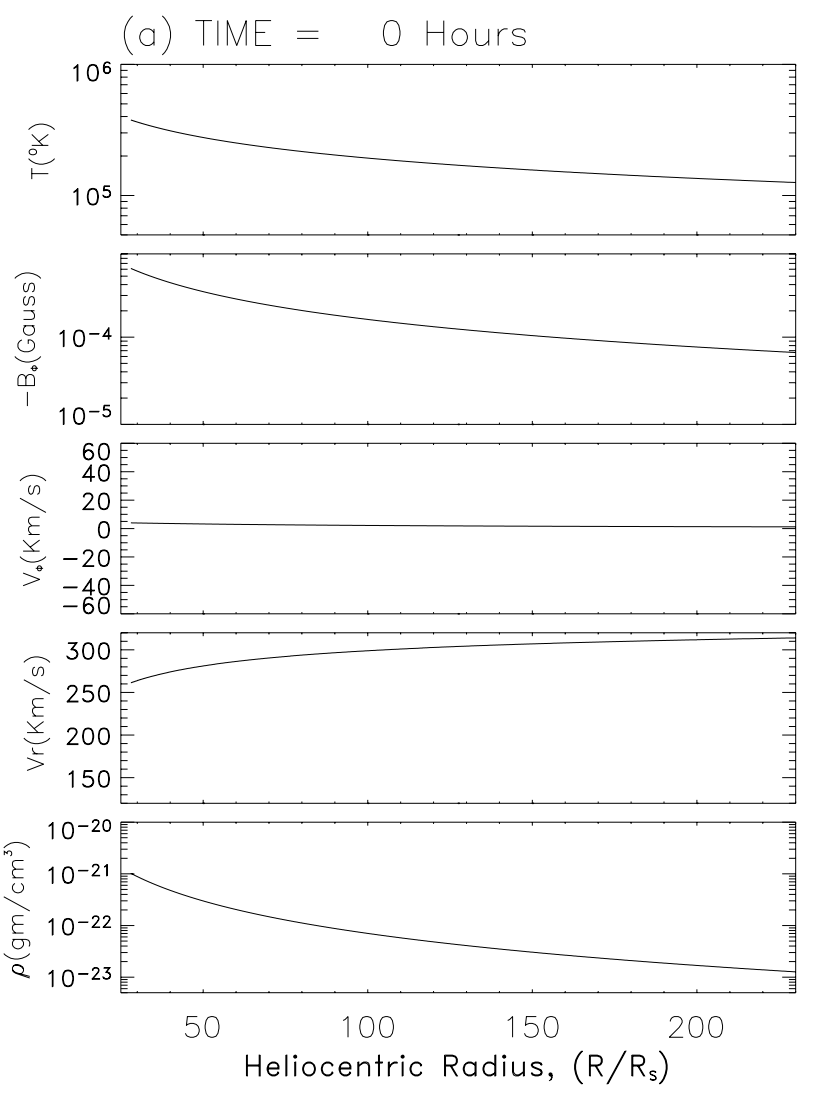

Fig. 1. The initial representative steady-state of the solar wind. Note that the $B_{\phi}$ direction is in the direction of a typical, Archimedean IMF outward polarity.

number of slow shocks were observed, we assume that the slow shock might be overtaken by the fast shock from behind. Therefore, based on the results of the above two cases, we put a perturbation of $\frac{v_{r}^{\prime}}{v_{r_{0}}}=0.4$ and $\frac{T^{\prime}}{T_{0}}=0.4$ at the begin$\operatorname{ning}(\mathrm{t}=0 \mathrm{~h})$. Then, we put another perturbation with $\frac{v_{r}^{\prime \prime}}{v_{r_{0}}}=1.6$ and $\frac{T^{\prime \prime}}{T_{0}}=5.0$ at $\mathrm{t}=15 \mathrm{~h}$ at the lower boundary, where " $v_{r}^{\prime \prime \prime}$ " and " $\mathrm{T}$ " " represent the temporal evolution of the second input pulses of temperature and radial velocity at $28 \mathrm{R}_{\odot}$. The rise and decay time of each disturbance is $1 \mathrm{~h}$, and the duration time is again $9 \mathrm{~h}$. We refer to this combined example as Case 3.

In Case 3, before we introduce a second perturbation (initiated at $\mathrm{t}=15 \mathrm{~h}$ ), a reverse slow shock (RSS) and a forward slow shock (FSS) are generated $15 \mathrm{~h}$ after the first perturbation was initiated. The results of first $15 \mathrm{~h}$ is the same as that shown in Case 1 (see Fig. 2a) just at the moment of the second pulse's initiation as noted above. We now look at this process in more detail. Figure 4 shows a set of high time resolution profiles of the evolutionary development of the complex set of discontinuities that are described as follows for Case 3. At $\mathrm{t}=21 \mathrm{~h}$ (see Fig. 4a for Case 3), a FSS(A) is located at about $41 \mathrm{R}_{\odot}$; a FFS(B) and FSS(B) follow it. "A" denotes the shock that was generated by the first perturbation, 


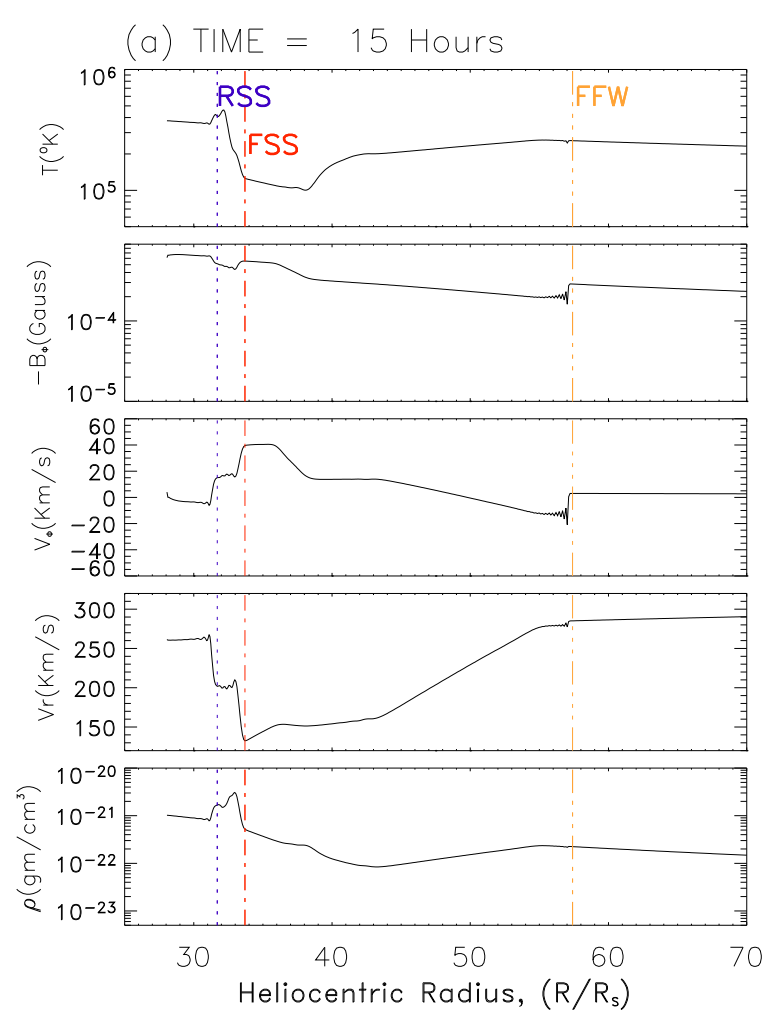

Fig. 2. Solar wind solution versus heliocentric distance for Case 1 heliocentric distance for $\mathrm{t}=15$ and $97 \mathrm{~h}$, respectively.

and "B" denotes the shock that was generated by the second perturbation (initiated at $\mathrm{t}=15 \mathrm{~h}$ ). At $\mathrm{t}=22.06 \mathrm{~h}$ (see Fig. 4b) the FFS(B) overtakes the FSS(A) from behind and appears to eliminate it temporarily. In Fig. 4c, at $\mathrm{t}=22.31 \mathrm{~h}$, the FSS(A) reappears behind the FFS(B). This $\operatorname{FSS}(\mathrm{A})$ merges with the other FSS(B) after it was overtaken by FFS(B) from behind. These two slow shocks merge into a nonlinear slow shocklike structure, SSLS (see Fig. 4d). This entire ensemble is trailed by a reverse fast shock, RFS(B). The reverse slow shock generated by the first perturbation (Case 1, Fig. 2) is not able to be identified.

Figure 5, in a continuation of temporal development in Case 3, shows the transition of the slow shock-like structure evolution in detail. This nonlinear wave is a slow shock-like structure (SSLS) (see Fig. 4d for $\mathrm{t}=22.8 \mathrm{~h}$ ), which evolves into an intermediate shock-like structure, ISLS (see Fig. 5a for $\mathrm{t}=23.3 \mathrm{~h}$ ). The magnetic field in the $\phi$-direction drops to a minimum at $\mathrm{t}=25.08 \mathrm{~h}$ (see Fig. 5b). The ISLS exists only for a short period (about $\sim 4 \mathrm{~h}$, see Figs. $5 \mathrm{~b}, \mathrm{c}, \mathrm{d}$ ), evolving into a SSLS (see Figs. 5c and d) and a nonlinear intermediate wave (IW, identified by a vertical long-dashed line). This ensemble is followed by the survival of a reverse fast shock, RFS(B) and a reverse slow wave, RSW. The SSLS evolved into a forward slow shock, FSS(A+B) (see Fig. 6). The nonlinear intermediate wave decayed and was not able to be identified after interacting with non-uniform solar wind (see Figs. 6c and d).

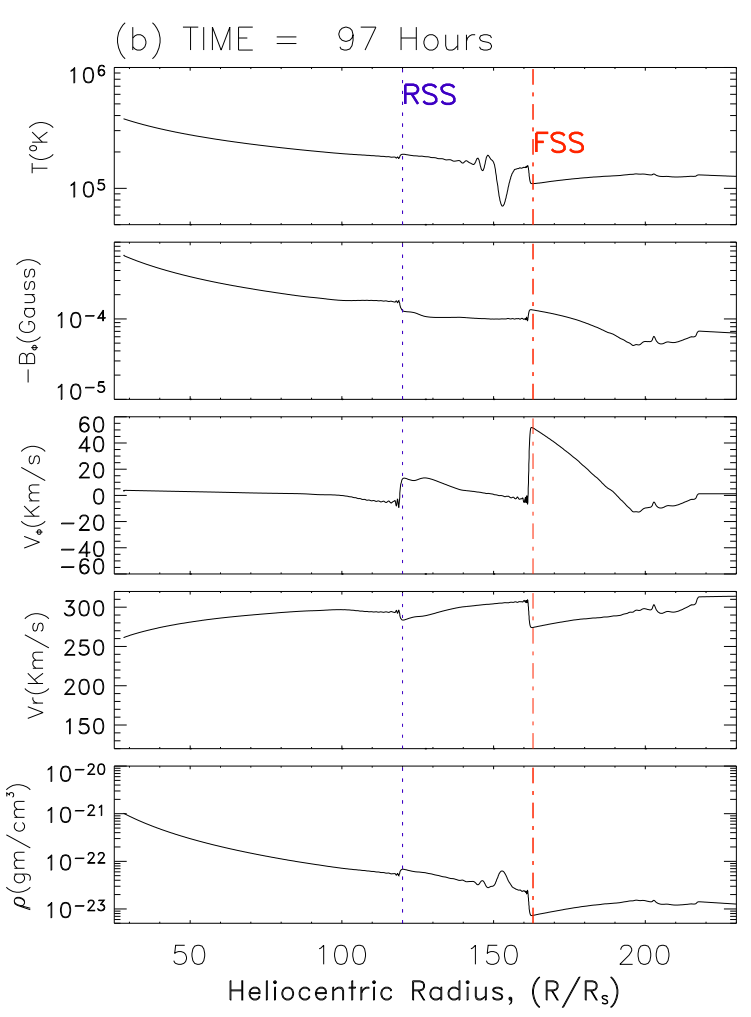

$\frac{v_{r}^{\prime}}{v_{r_{\mathrm{o}}}}=0.4, \frac{T^{\prime}}{T^{\circ}}=0.4$. (a) and (b) show the solar wind solution versus

\section{Discussion and Summary}

The purpose of this study is intended to be an exploratory examination of numerical studies of shock interaction in the solar wind; we made no attempt to perform a parametric study. In the first case of numerical simulation, a pair of slow shocks (one FSS and one RSS) was generated by a perturbation at $\mathrm{r}=28 R_{\odot}$, consisting of a combined decrease of pressure and radial velocity. In the second case of numerical simulation, a forward fast shock and a forward slow shock were generated by a perturbation consisting of an increase in both pressure and radial velocity. Physically, these two cases may be considered to be a combination of momentum and pressure decreases (Case 1) or increases (Case 2).

The perturbations for Cases 1 and 2 were then combined to be Case 3, in order to examine in detail the evolution of a fast-slow shock interaction. In this latter case, the slow shock disappeared temporarily but then reappeared after the fast shock overtook and passed through the slow shock. At the moment of disappearance of the slow shock, it seemed that it was destroyed by the fact that it had been overtaken by the fast shock. The merging of two slow shocks appears, at first, to produce another slow shock; however, the evolution of the merging of these two slow shocks generates other forms of discontinuities, namely shocks and waves. In the beginning of this merging, a slow shock-like structure (SSLS) was created. A SSLS was temporarily created, evolving into an 

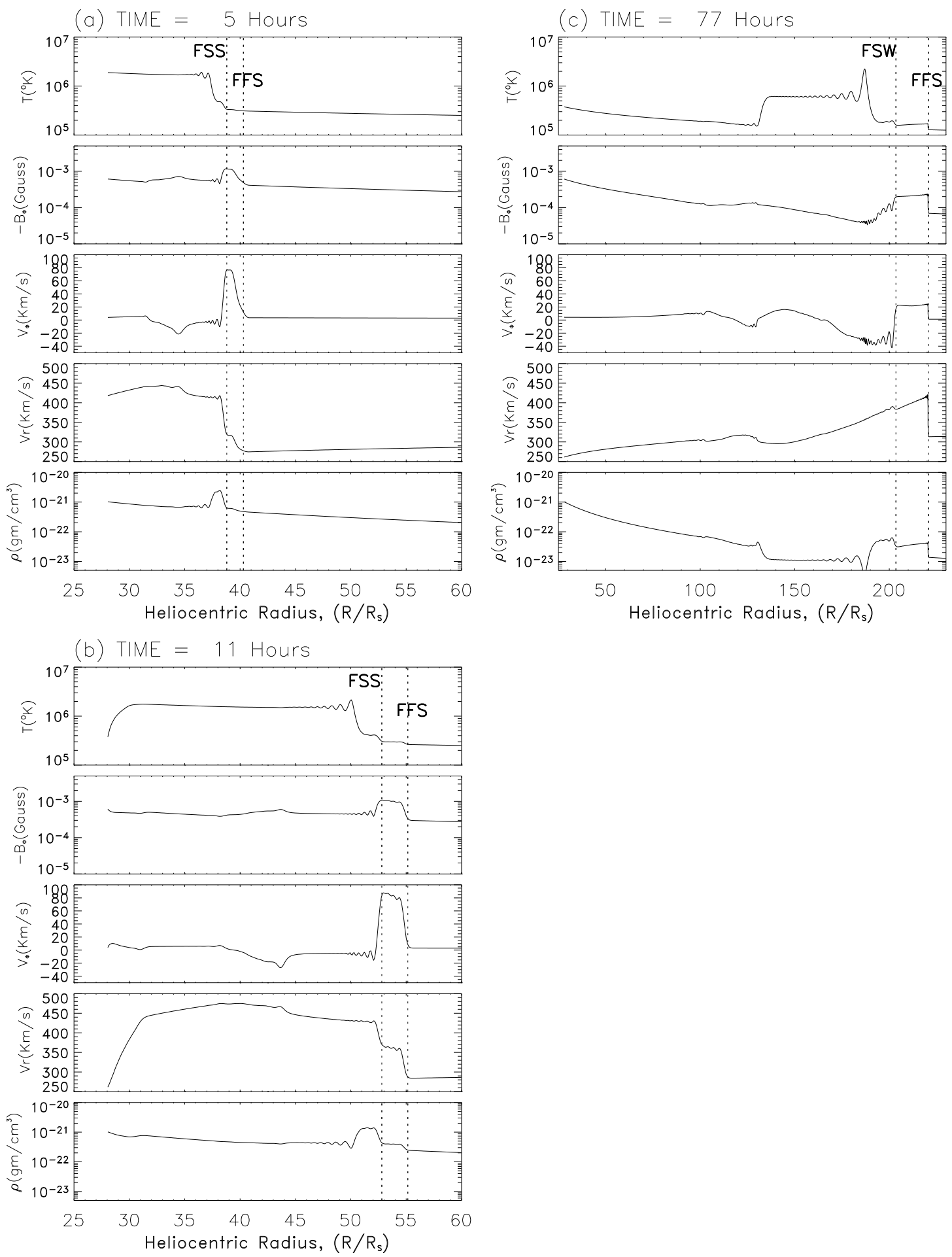

Fig. 3. Solar wind solution versus heliocentric distance for Case $2: \frac{v_{r}^{\prime}}{v_{r_{0}}}=1.6, \frac{T^{\prime}}{T^{\circ}}=5.0$. (a), (b) and (c) show the solar wind solution versus heliocentric distance for $\mathrm{t}=5,11$ and $77 \mathrm{~h}$. Note that non-physical oscillations linked to the use of the Lax-Wendroff scheme are found behind the fast slow wave, FSW.

intermediate shock-like structure (ISLS). This latter structure existed for only $\sim 4$ hours, evolving again into another slow shock plus an intermediate wave; this latter wave, itself, disappeared via interaction with the upstream non-uniform solar wind. Our Case 3 then demonstrated the extremely complex set of discontinuities by changing the set of boundary conditions which may relate to physically-changing solar boundary conditions. We have not compared our simulations to 

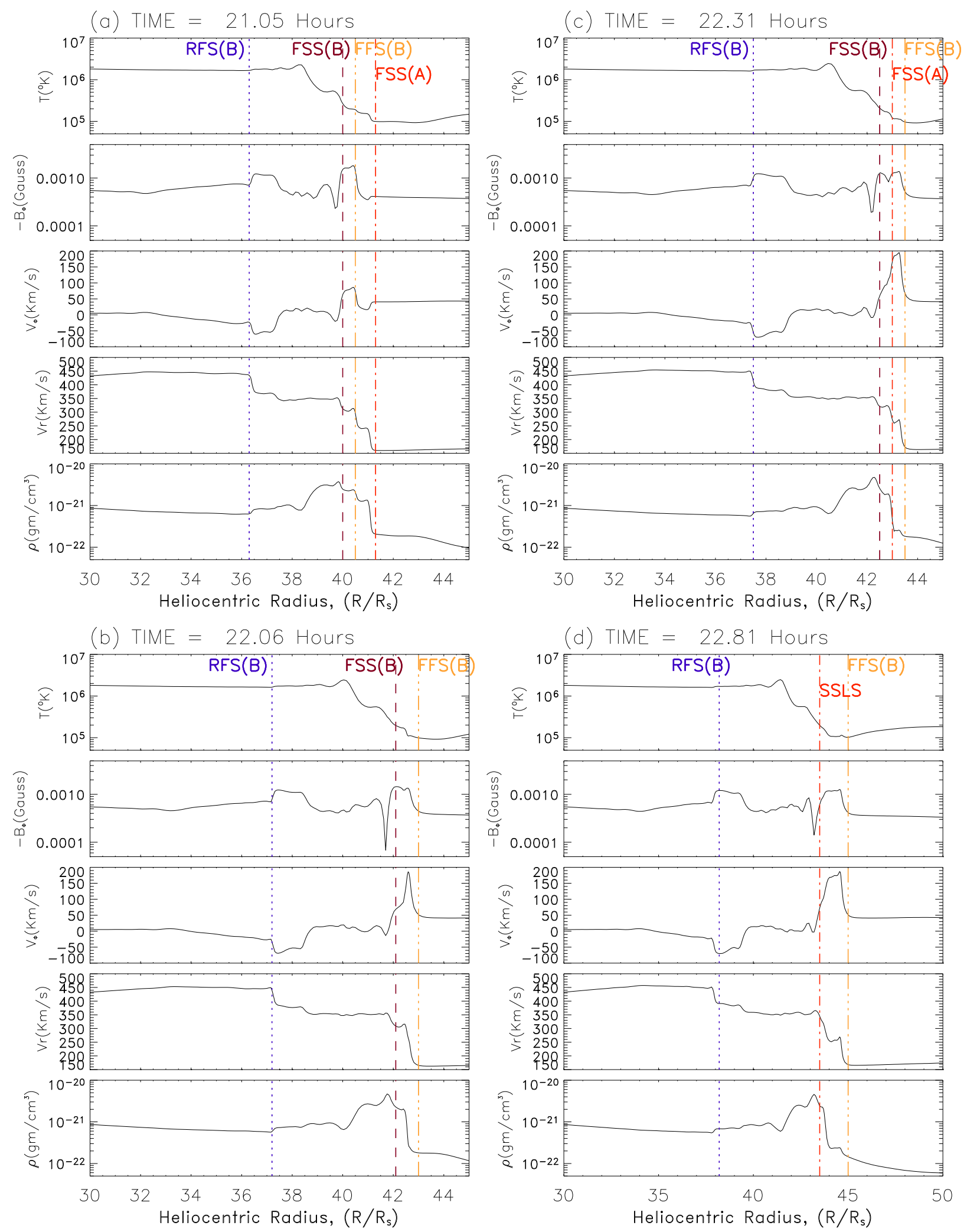

Fig. 4. Solar wind solution versus heliocentric distance for Case 3: $\frac{v_{r}^{\prime}}{v_{r_{0}}}=0.4, \frac{T^{\prime}}{T^{\circ}}=0.4 \frac{v_{r}^{\prime \prime}}{v_{r_{0}}}=1.6, \frac{T^{\prime \prime}}{T^{\circ}}=5.0$. (a), (b), (c) and (d) show the solar wind solution versus heliocentric distance for $\mathrm{t}=21.05,22.06,22.31$ and $22.81 \mathrm{~h}$, respectively. 

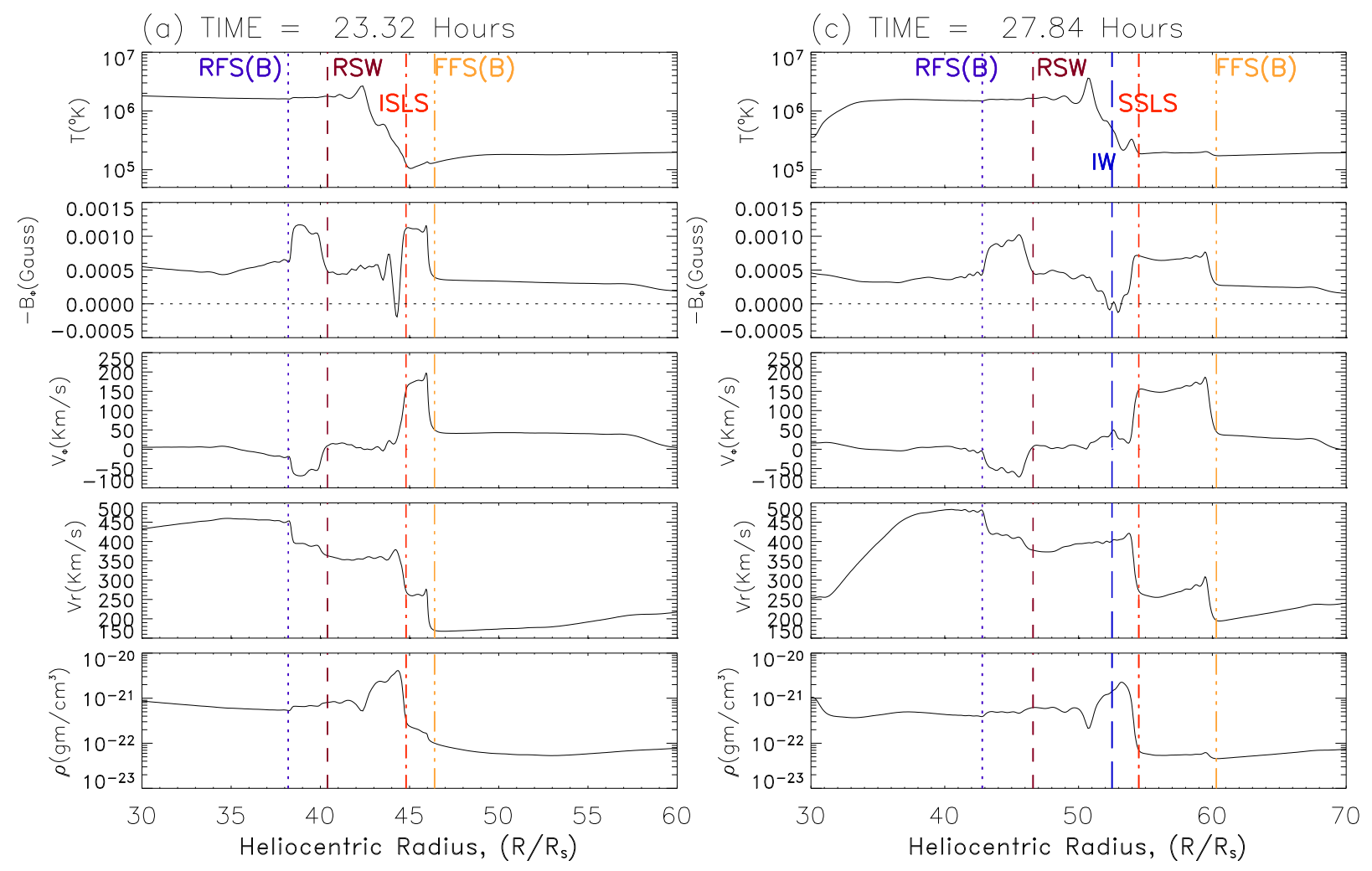

(b) TIME $=25.08$ Hours
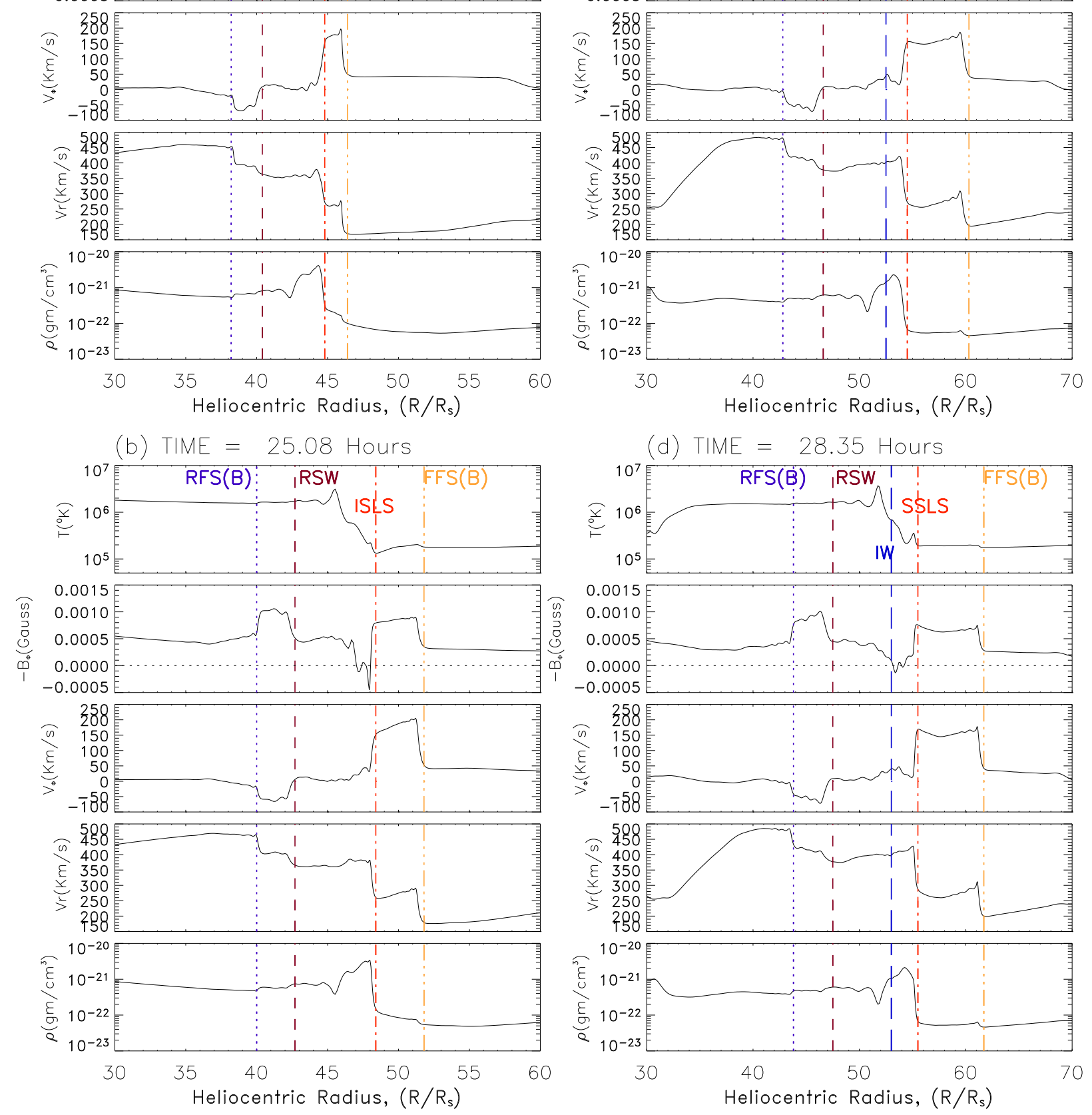

(d) TIME $=28.35$ Hours
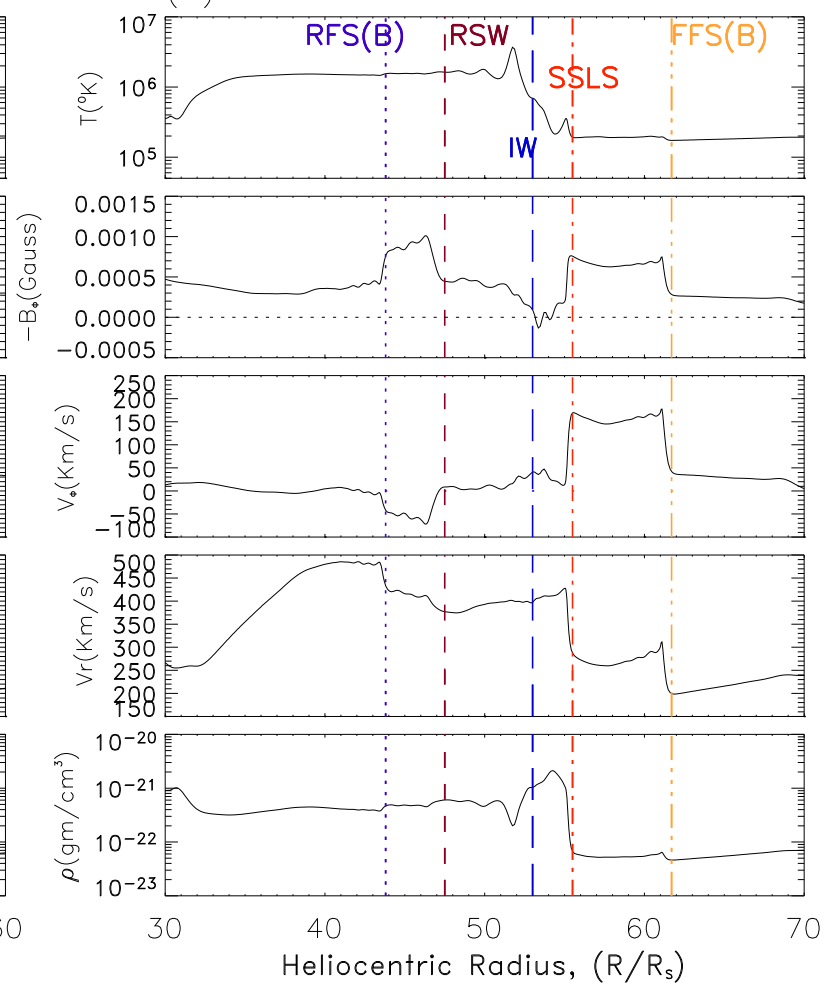

Fig. 5. Solar wind solution versus heliocentric distance for Case 3: $\frac{v_{r}^{\prime}}{v_{r_{0}}}=0.4, \frac{T^{\prime}}{T^{\circ}}=0.4 \frac{v_{r}^{\prime \prime}}{v_{r_{0}}}=1.6, \frac{T^{\prime \prime}}{T^{\circ}}=5.0$. (a), (b), (c) and (d) show the solar wind solution versus heliocentric distance for $\mathrm{t}=23.32,25.08,27.84$ and $28.35 \mathrm{~h}$, respectively. Note that the RSW represents a reverse slow wave and IW represents an intermediate wave. 

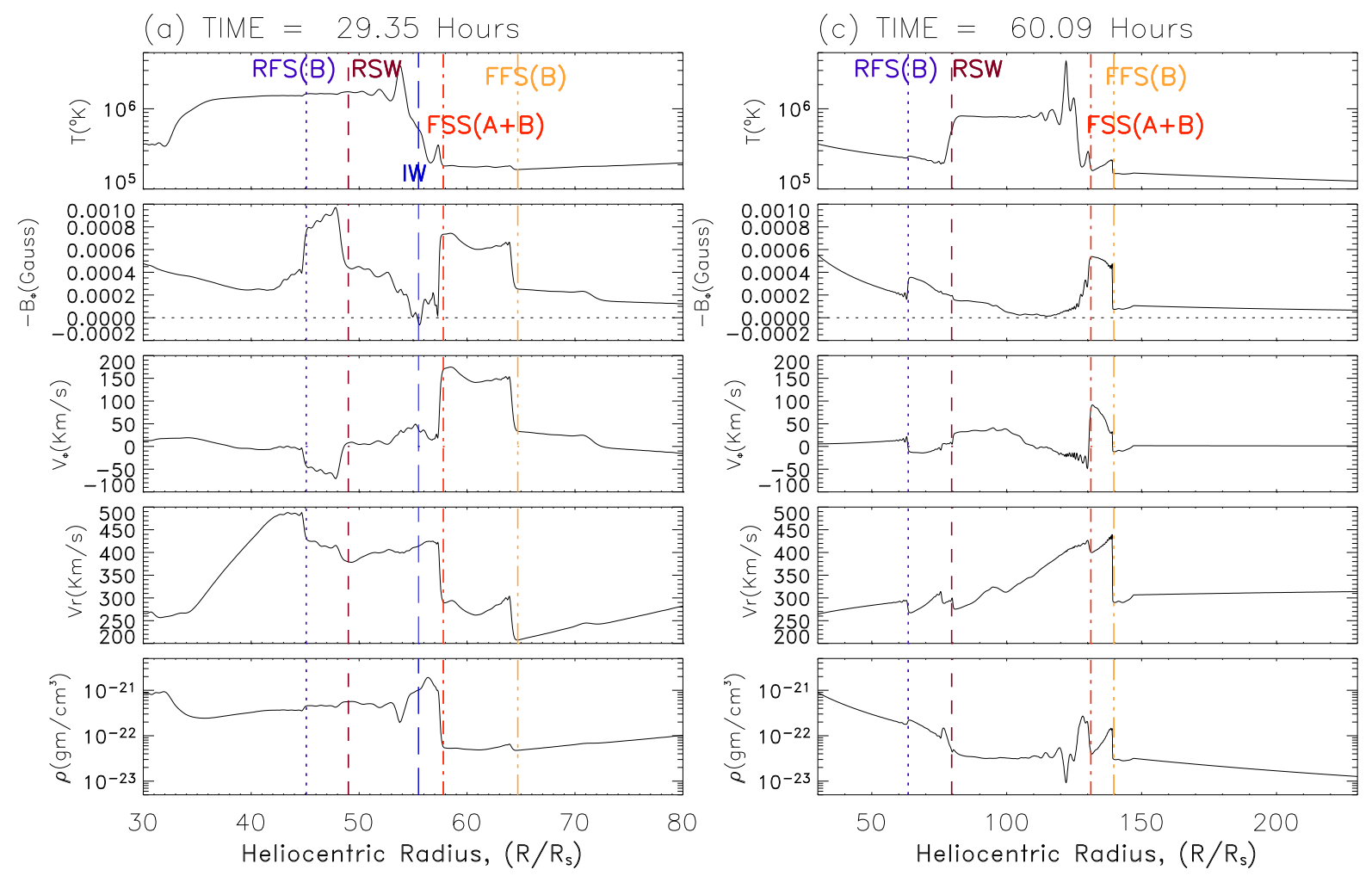

(b) TIME $=30.11$ Hours

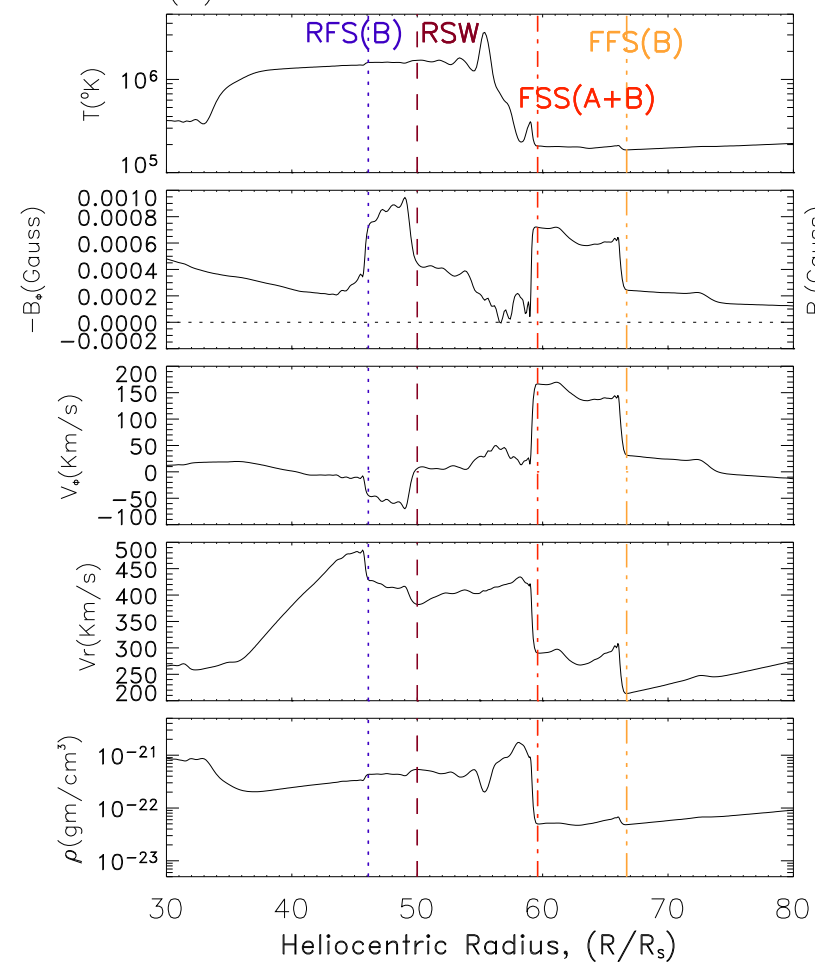

(d) TIME $=93.08$ Hours
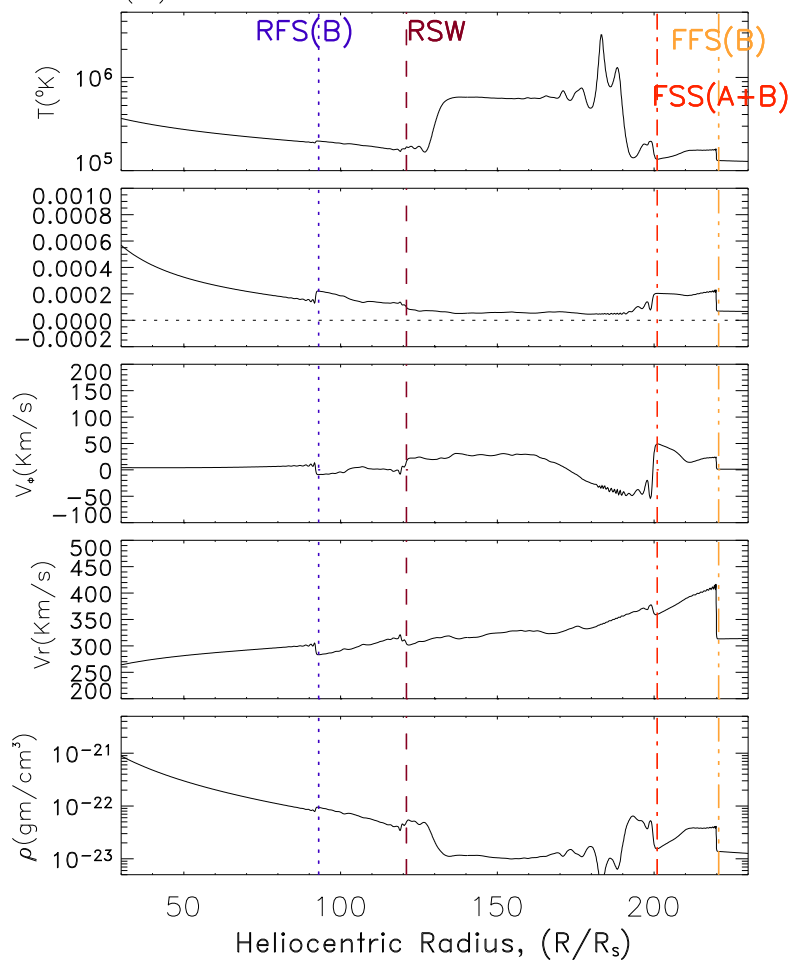

Fig. 6. Solar wind solution versus heliocentric distance for Case 3: $\frac{v_{r}^{\prime}}{v_{r_{0}}}=0.4, \frac{T^{\prime}}{T^{\circ}}=0.4 \frac{v_{r}^{\prime \prime}}{v_{r_{0}}}=1.6, \frac{T^{\prime \prime}}{T^{\circ}}=5.0$. (a), (b), (c) and (d) show the solar wind solution versus heliocentric distance for $\mathrm{t}=29.35,30.11,60.09$ and $93.08 \mathrm{~h}$, respectively. Note that the RSW represents a reverse slow wave and IW represents an intermediate wave. 

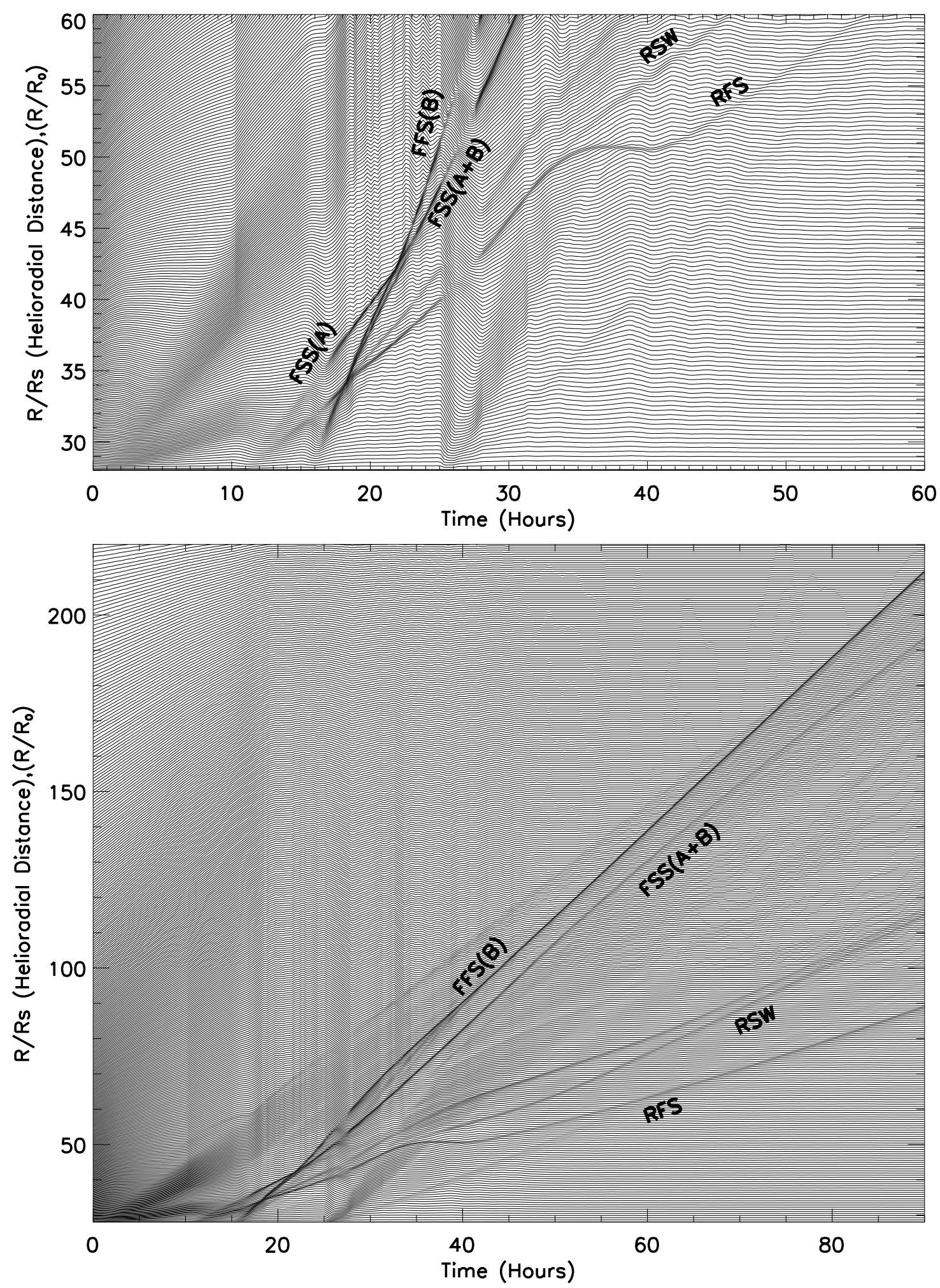

Fig. 7. Radius-time profile for Case 3 . The upper panel shows the details, from the lower boundary to $60 R_{\odot}$, of the various discontinuities (see text), as well as the adaptive grid's ability for fine tuning the grid sizes. The lower panel shows the continuation of these discontinuities to larger distances. 
observations, since that objective is beyond the scope of the present investigation.

For the SSLS, the density, radial velocity and temperature increase, and the transverse component of the magnetic field decreases. However, the jump conditions do not satisfy the Rankine-Hugoniot relations. Therefore, we call it an SSLS instead of a slow shock.

For the ISLS, the density, radial velocity, and temperature increase; the transverse component of the magnetic field of the ISLS changes direction, but the Alfveen Mach number is less than one. Therefore, we call it an ISLS instead of an intermediate shock. The procedure of the shock strength Mach number calculation method is based strictly on steadystate mass flux through the shock, regardless of its type (fast, slow, intermediate, etc.). We also used a straightforward method of calculating the average shock Mach number between two radial positions as the shock propagating from one position at time, $t_{1}$ (say) to a second position at $t_{2}$. We call this latter procedure the Wave Transit Method (WTM). The detailed computational procedure for WTM is given by Wu et al. (1996a,b). (We used higher resolution data to carefully identify the upstream location of ISLS for each time step. Only a single point data is used.)

The intermediate shock-like structure evolves into a slow shock-like structure and an intermediate wave. The slow shock-like structure steepened into a slow shock, but the intermediate wave disappeared by interaction with the nonunique solar wind. This slow shock does not evolve into any other type of shock or discontinuity.

The grid location is changing all the time for the adaptive grid model used. The grid resolution is higher around the area with higher velocity gradient (e.g. around the shocks). It helps to identify the waves' location by seeing the grids' histogram for the entire simulation result. For example, Fig. 7 shows the radius-time profile for Case 3. The top panel is the enlargement of the bottom panel near the Sun. The vertical axis shows the radial distance and the horizontal axis shows the time series. The location of major waves (e.g., FFS, FSS, RFS, RSW) are clearly shown. The interaction between the fast and slow shock waves are also clear, as shown in the top panel of Fig. 7.

In summary, the forward slow shock and the reverse slow shock will be generated by a perturbation with a deceleration of solar wind. A forward slow shock is not destroyed by being overtaken by a forward fast shock. However, the evolution of this interaction shows that the slow shock might disappear temporarily, but it will reappear after the fast shock interacts with the slow shock. The present simulation also shows that the merging of two forward slow shocks will create another stronger slow shock. However, during the evolution of the two merging slow shocks, a slow shocklike structure is first developed. Then this slow shock-like structure evolves into an intermediate shock-like structure. Later, the intermediate shock-like structure evolves into a slow shock-like structure and an intermediate wave. Finally, the intermediate wave disappears, and the slow shock-like structure evolves into a slow shock by interacting with the non-uniform solar wind. This slow shock does not evolve into any other type of shock or discontinuity.

Finally, we note that this limited study of slow shock interactions was made possible by the development of the adaptive grid numerical technique. The results described above are not unique; instead, they are representative of a wide class of solutions that may be generated by a variety of physicallybased changes in solar conditions.

As a result of this non-uniqueness, one might safely conclude by noting that, if a complex set of discontinuities were to be observed by a spacecraft, a correct inference of the responsible boundary conditions at the Sun would (except for simple cases) be impossible.

Acknowledgements. The work of CCW is supported partially by the NASA Living with a Star Targeted R\&D program, NASA Grant (NAG-12527 and NAG-512467) and Space Weather Program of National Science Foundation, ATM-028414. STW wishes to acknowledge support of this work via National Science Foundation (NSF) Grant ATM0310115 and NASA Grant NAG5-12843. MD also acknowledges support of a Living With a Star Targeted R\&D Program, NASA Grant NAG-12527, and the hospitality of the NOAA Space Environment Center. The authors thank both referees for their constructive suggestions.

Topical Editor R. Forsyth thanks A. Usmanov and another referee for their help in evaluating this paper.

\section{References}

Chao, J. K., Wu, S. T., and Wu, C.-C.: Evolution of slow shocks in the solar wind, Proceedings of the Sixth International Solar Wind Conference, YMCA of the Rockies, Estes Park, Colorado, U.S.A., August 23-28, 1987.

Chao, J. K., Lyu, L. H., Wu, B. H., Lazarus, A. J., Chang, T. S., and Lepping, R. P.: Observations of an intermediate shock in interplanetary space, J. Geophys. Res., 98, 17 443-17 450, 1993.

Chu, C. K. and Taussig, R. T.: Numerical experiments of magnetohydrodynamic shocks and the stability of switch-on shocks, The Physics of Fluids, 10, 249-256, 1967.

DeHoffmann, F., and E. Teller, Phys. Rev. 80, 692-703, 1950.

Hughes, W. F. and Brighton, J. A.: Fluid Dynamics, Schaum's Outline Series, Mac Graw-Hill Book Company, New York, 1967.

Kennel, C. F., Blandford, R. D., and Coppi, P.: MHD intermediate shock discontinuities, Part 1, Rankine-Hugoniot conditions, J. Plasma Phys., 42, 299-319, 1989.

Lax, P. D. and Wendroff, B.: Systems of conservation laws, Comm. Pure. and Appl. Math., 13, 217-237, 1960.

Panitchob, S.: An adaptive grid finite difference method for timedependent Magnetohydrodynamic (MHD) flows and its astrogeophysical applications, Ph.D. thesis, The department of Mechanical Engineering, The University of Alabama in Huntsville, 1987.

Panitchob, S., Wu, S. T., and Suess, S. T.: An adaptive grid unsteady model for two-dimensional magnetodrodynamic (MHD) flow, AIAA-87-1218, American Inst. of Aeronautics and Astronautics, Washington, D.C., 1987.

Priest, E. R.: Solar Magnetohydrodynamics, D. Reidel Publishing Company, Dordrecht, Holland, 1982.

Whang, Y. C.: Slow shocks and their transition to fast shocks, J. Geophys. Res., 92, 4349-4356, 1987. 
Wu, C. C.: MHD intermediate shocks, Geophys. Res. Lett., 14, 377-393, 1987.

Wu, C.-C.: Numerical simulation of interplanetary dynamics, Ph.D. thesis, The University of Alabama in Huntsville, 1993.

Wu, C. C., Chao, J. K., Wu, S. T., and Dryer, M.: Numerical simulation interplanetary slow shocks in the solar wind, Solar Phys., 165, 377-393, 1996a.
Wu, C. C., Wu, S. T. and Dryer, M.: Generation and evolution of interplanetary slow shock, Ann. Geophys., 14, 375-382, 1996b, SRef-ID: 1432-0576/ag/1996-14-375.

Wu, S. T., and Wang, J. F.: Numerical tests of a modified full implicit continuous Eulerian (FICE) scheme with projected normal characteristic boundary conditions for MHD flows, Comp. Meth. in Appl. Mech. and Eng., 64, 267-282, 1987. 